\title{
An Explicit SU(12) Family and Flavor Unification Model
}

\author{
Carl H. Albright ${ }^{* a, b}$ Robert P. Feger, ${ }^{c}$ and Thomas W. Kephart ${ }^{c}$ \\ ${ }^{a}$ Department of Physics, Northern Illinois University \\ DeKalb, IL 60115, USA \\ ${ }^{b}$ Theoretical Physics, Fermilab \\ Batavia, IL 60510, USA \\ ${ }^{c}$ Department of Physics and Astronomy, Vanderbilt University \\ Nashville, TN 37235, USA \\ E-mail: albrightefnal.gov, robert.feger@vanderbilt.edu, \\ tom.kephart@gmail.com
}

\begin{abstract}
An explicit SUSY SU(12) unification model with three light chiral families is presented which avoids any external flavor symmetries. The hierarchy of quark and lepton masses and mixings is explained by higher dimensional Yukawa interactions involving Higgs bosons containing SU(5) singlet fields with VEVs appearing at or below the SUSY GUT scale of $2 \times 10^{16} \mathrm{GeV}$, approximately 50 times smaller than the SU(12) unification scale. The model has been found to be in good agreement with the observed quark and lepton masses and mixings, with nearly all prefactors of $\mathscr{O}(1)$ in the four Dirac and one Majorana fermion mass matrices.
\end{abstract}

36th International Conference on High Energy Physics,

July 4-11, 2012

Melbourne, Australia

${ }^{*}$ Speaker. 


\section{Motivation}

Grand Unified Theories (GUTs) of quarks and leptons have played an important role for almost 40 years in theoretical attempts to make sense of the apparent group structure and mass spectra of the quark and lepton matter fields and coupling strengths of the gauge and Higgs fields observed in Nature. But among the most popular groups, the only appropriate chiral representations (irreps) are $\mathbf{1 0}, \overline{\mathbf{5}}$, and $\mathbf{1}$ for $\mathrm{SU}(5), \mathbf{1 6}$ for $\mathrm{SO}(10)$, and $\mathbf{2 7}$ for $\mathrm{E}_{6}$. In order to accommodate the three families observed todate, it has been conventional to introduce in addition to one of the above $G_{\text {family }}$ groups, a $G_{\text {flavor }}$ symmetry group which also distinguishes the families. The flavor group chosen may be continuous or discrete, so in either case the GUT model then involves the direct product group $G_{\text {family }} \times G_{\text {flavor }}$.

True family and flavor unification requires the introduction of a higher rank simple group. Some earlier studies along this line have been based on enlarged groups such as $\mathrm{SO}(18), \mathrm{SU}(8)$, $\mathrm{SU}(9)$, and $\mathrm{SU}(11)$, but none have been totally satisfactory due to a huge number of unwanted states and/or unsatisfactory mass matrices. Here we describe an SU(12) unification model [1] with interesting features that was constructed with the help of a Mathematica computer package called LieART written by two of us (RPF and TWK) [2]. This program allows one to compute tensor products, branching rules, etc., and perform detailed searches for satisfactory models in a timely fashion. While other smaller and larger rank unitary groups were examined, a model based on $\mathrm{SU}(12)$ appeared to be the most satisfactory minimal one for our purpose. We sketch here the model construction and point out that further details and references can be found in [1] and [3].

\section{SU(12) Unification Model and Particle Assignments}

The unification group SU(12) has 11 totally antisymmetric irreps, of which 10 are complex which allow three $\mathrm{SU}(5)$ families to be assigned to different chiral irreps. For this purpose, one can choose an anomaly-free set of SU(12) irreps which contains three chiral SU(5) families and pairs of fermions which will become massive at the SU(5) scale. One such suitable set consists of

$$
6(\mathbf{4 9 5})+4(\overline{\mathbf{7 9 2}})+4(\overline{\mathbf{2 2 0}})+(\overline{\mathbf{6 6}})+4(\overline{\mathbf{1 2}}) \rightarrow 3(\mathbf{1 0}+\overline{\mathbf{5}}+\mathbf{1})+238(\mathbf{5}+\overline{\mathbf{5}})+211(\mathbf{1 0}+\overline{\mathbf{1 0}})+484(\mathbf{1})
$$

where the decomposition to anomaly-free $\mathrm{SU}(5)$ states has been indicated.

With this choice a search through the possible assignments of the three light chiral families to the SU(12) irreps appearing in the anomaly-free set of Eq. (1) reveals the following selection for a satisfactory low scale phenomenology:

$$
\begin{aligned}
& \text { 1st Family: }(\mathbf{1 0}) \mathbf{4 9 5}_{\mathbf{1}} \supset u_{L}, u_{L}^{c}, d_{L}, e_{L}^{c} \\
& (\overline{\mathbf{5}}) \overline{\mathbf{6 6}_{\mathbf{1}}} \supset d_{L}^{c}, e_{L}, v_{1 L} \\
& \text { (1) } \overline{\mathbf{7 9 2}} \supset N_{1 L}^{c} \\
& \text { 2nd Family: (10) } \overline{\mathbf{7 9 2}} \supset c_{L}, c_{L}^{c}, s_{L}, \mu_{L}^{c} \\
& \left(\overline{\mathbf{5}} \overline{\mathbf{7 9 2}} \supset s_{L}^{c}, \mu_{L}, v_{2 L}\right. \\
& \text { (1) } \overline{\mathbf{2 2 0}_{2}} \supset N_{2 L}^{c} \\
& \text { 3rd Family : }(\mathbf{1 0}) \overline{\mathbf{2 2 0 _ { 3 }}} \supset t_{L}, t_{L}^{c}, b_{L}, \tau_{L}^{c} \\
& (\overline{\mathbf{5}}) \overline{\mathbf{7 9 2}} \supset b_{L}^{c}, \tau_{L}, v_{3 L} \\
& \text { (1) } \overline{\mathbf{1 2}} \supset N_{3 L}^{c}
\end{aligned}
$$


Here the subscripts on the $\mathrm{SU}(12)$ irreps refer to the family in question, while the numbers in parentheses are just the $\mathrm{SU}(5)$ irreps chosen. Note that each $\mathrm{SU}(5)$ family multiplet can be uniquely assigned to a different SU(12) multiplet in the anomaly-free set according to (1). On the other hand, the remaining $\mathrm{SU}(5)$ multiplets are unassigned but form conjugate pairs which become massive and decouple at the $\mathrm{SU}(5)$ scale and are of no further interest.

In order to pursue an effective theory approach based on leading order tree diagrams, we start with the SU(12) model sketched above and take it to be supersymmetric. With an appropriate set of Higgs fields, the SU(12) symmetry can be broken down to SU(5) while preserving supersymmetry [4,5]. Unbroken supersymmetry at the SU(5) GUT scale allows us to deal only with tree diagrams in order to generate higher dimensional operators, for loop corrections are much suppressed.

For this purpose, we introduce massive $\mathbf{2 2 0} \times \overline{\mathbf{2 2 0}}$ and $\mathbf{7 9 2} \times \overline{\mathbf{7 9 2}}$ fermion pairs at the SU(12) scale. In addition, we introduce $(\mathbf{1}) \mathbf{6 6}_{\mathbf{H}},(\mathbf{1}) \overline{\mathbf{6 6}_{\mathbf{H}}}$, and $(\mathbf{1}) \mathbf{2 2 0}_{\mathbf{H}},(\mathbf{1}) \overline{\mathbf{2 2 0 _ { \mathbf { H } }}}$ conjugate Higgs pairs which acquire SU(5) singlet VEVs at the SUSY SU(5) GUT scale. Finally, doublets in $(\mathbf{5}) \mathbf{9 2 4}_{\mathbf{H}}$ and $(\overline{\mathbf{5}}) \mathbf{9 2 4}_{\mathbf{H}}$ Higgs fields effect the electroweak symmetry breaking at the electroweak scale. For each element of the quark and lepton mass matrices, tree diagrams can then be constructed from three-point vertices which respect the $\mathrm{SU}(12)$ and $\mathrm{SU}(5)$ multiplication rules.

For illustration we present the lowest order tree diagram contributions to the 33 elements taking into account the family assignments in (2.2) for the up and down quark mass matrices, as well as the Dirac and Majorana neutrino mass matrices . These are listed as U33, D33, DN33, and MN33, respectively:

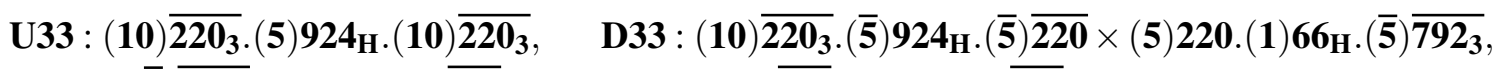

$$
\begin{aligned}
& \text { DN33 : }(\overline{\mathbf{5}}) \overline{\mathbf{7 9 2}_{3}} \cdot(\mathbf{5}) \mathbf{9 2 4}_{\mathrm{H}} \cdot(\mathbf{1}) \overline{\mathbf{1 2}_{3}}, \quad \mathrm{MN33}:(\mathbf{1}) \overline{\mathbf{1 2}_{3}} \cdot(\mathbf{1}) 6_{\mathrm{H}} \cdot(\mathbf{1}) \overline{12_{3}} \text {. }
\end{aligned}
$$

The convention is adopted that the left-handed fields appear on the left and the left-handed conjugate fields appear on the right. The dots indicate the beginning or ending of a three-point vertex, while the $\times$ refers to a fermion mass insertion. The leading order terms for $\mathbf{U 3 3}, \mathbf{D N 3 3}$, and $\mathbf{M N 3 3}$ are seen to have dim-4, while that for D33 has dim-5, due to the $\mathbf{1}_{\mathbf{1}} \mathbf{6 6}_{\mathbf{H}} \mathrm{SU}(5)$ Higgs singlet insertion resulting in one extra external Higgs field. The corresponding diagram for the charged lepton 33 mass matrix element is just the transpose of $\mathbf{D 3 3}$.

In a similar fashion one can obtain the full set of leading-order diagrams for each matrix element of the five mass matrices. It is rather remarkable that only one diagram for each matrix element appears at leading order for all five mass matrices. Again we refer the interested reader to references [1,3] for the details.

\section{Mass Matrices and Mixings}

With the leading-order diagrams for each matrix element in hand, we can then construct the quark and lepton mass matrices as follows. To each diagram corresponds a coupling constant or prefactor, $h_{i j}^{u}, h_{i j}^{d}, h_{i j}^{d n}$ or $h_{i j}^{m n}$ for the $i j$ th element of the appropriate mass matrix, which is assumed to be of order one at the SU(12) unification scale, as naturalness predicts. Every SU(5) Higgs singlet insertion in higher-order tree diagrams introduces one power of $\varepsilon \equiv M_{\mathrm{SU}(5)} / M_{\mathrm{SU}(12)} \sim 1 / 50$ 
through the appearance of the ratio of the singlet Higgs VEV to the mass of the conjugate fermion fields after the latter are integrated out. Finally as a result of the electroweak spontaneous symmetry breaking, the $\mathbf{9 2 4}_{\mathbf{H}}$ acquires a weak scale VEV, v. The resulting mass matrices then obtained are the following:

$$
\begin{aligned}
M_{U} & =\left(\begin{array}{lll}
h_{11}^{u} \varepsilon^{4} & h_{12}^{u} \varepsilon^{3} & h_{13}^{u} \varepsilon^{2} \\
h_{12}^{u} \varepsilon^{3} & h_{22}^{u} \varepsilon^{2} & h_{23}^{u} \varepsilon \\
h_{13}^{u} \varepsilon^{2} & h_{23}^{u} \varepsilon & h_{33}^{u}
\end{array}\right) \mathrm{v}, \quad M_{D}=\left(\begin{array}{ccc}
h_{11}^{d} \varepsilon^{4} & h_{12}^{d} \varepsilon^{3} & h_{13}^{d} \varepsilon^{3} \\
h_{21}^{d} \varepsilon^{3} & h_{22}^{d} \varepsilon^{2} & h_{23}^{d} \varepsilon^{2} \\
h_{31}^{d} \varepsilon^{2} & h_{32}^{d} \varepsilon & h_{33}^{d} \varepsilon
\end{array}\right) \mathrm{v}, \\
M_{D N} & =\left(\begin{array}{ccc}
h_{11}^{d n} \varepsilon^{3} & h_{12}^{d n} \varepsilon^{2} & h_{13}^{d n} \varepsilon \\
h_{21}^{d n} \varepsilon^{2} & h_{22}^{d n} \varepsilon & h_{23}^{d n} \\
h_{31}^{d n} \varepsilon^{2} & h_{32}^{d n} \varepsilon & h_{33}^{d n}
\end{array}\right) \mathrm{v}, M_{M N}=\left(\begin{array}{ccc}
h_{11}^{m n} & h_{12}^{m n} \varepsilon & h_{13}^{m n} \varepsilon^{2} \\
h_{12}^{m n} \varepsilon & h_{22}^{m n} \varepsilon^{2} & h_{23}^{m n} \varepsilon^{3} \\
h_{13}^{m n} \varepsilon^{2} & h_{23}^{m n} \varepsilon^{3} & h_{33}^{m n}
\end{array}\right) \Lambda_{R} .
\end{aligned}
$$

Here $\Lambda_{R}$ represents the right-handed mass scale, typically of $\mathscr{O}\left(10^{14}\right) \mathrm{GeV}$, whereas the $\mathrm{SU}(5)$ SUSY GUT scale is $2 \times 10^{16} \mathrm{GeV}$ to obtain gauge coupling unification. Again, a factor of $\varepsilon$ enters for every singlet Higgs insertion in higher order diagrams. While $M_{U}$ and $M_{M N}$ are symmetric, $M_{D}$, $M_{L}=M_{D}^{T}$, and $M_{D N}$ are doubly lopsided in that the terms with $h_{23}^{d}, h_{32}^{l}$ and $h_{32}^{d n}$ are suppressed by one extra power of $\varepsilon$ compared to those with $h_{32}^{d}, h_{23}^{l}$, and $h_{23}^{d n}$, respectively; this is likewise true for the corresponding 13 and 31 terms. For $M_{D}$, this implies that a larger right-handed rotation than left-handed rotation is needed to bring the down quark matrix into diagonal form, while the opposite is true for $M_{L}$ and $M_{D N}$.

The symmetric light-neutrino mass matrix is obtained via the Type I seesaw mechanism:

$$
M_{v}=-M_{\mathrm{DN}} M_{\mathrm{MN}}^{-1} M_{\mathrm{DN}}^{T} .
$$

Keeping only the leading-order terms in $\varepsilon$ for each matrix element, we find

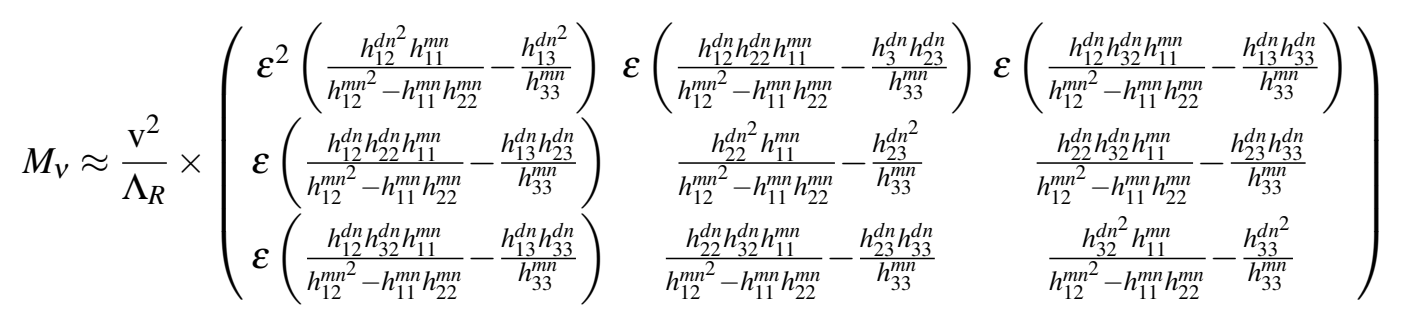

which does not involve the prefactors $h_{11}^{d n}, h_{21}^{d n}, h_{31}^{d n}, h_{13}^{m n}$ and $h_{23}^{m n}$.

The light-neutrino mass matrix exhibits a much milder hierarchy compared to the up-type and down-type mass matrices, as can be seen from the pattern of powers of $\varepsilon$. A mild or flat hierarchy of $M_{v}$ is conducive to obtaining large mixing angles and similar light neutrino masses.

\section{Numerical Results}

To obtain numerical results for the model predictions, we evaluate the mass matrices at the top quark mass scale and use just real prefactors to avoid too many fit parameters for good fit convergence. We refer the reader to our published paper [1] for full details of the fitting procedure, where we have included the latest best value for the reactor neutrino mixing angle, $\theta_{13}$. There can be found a table giving the phenomenological mass and mixing data entering the fit, as well as the theoretical mass and mixing results obtained from the fitting procedure. 
The best fit was obtained with a normal neutrino mass hierarchy with $\Lambda_{R}=7.4 \times 10^{14} \mathrm{GeV}$ and the following mass matrices:

$$
\begin{aligned}
& M_{U}=\left(\begin{array}{ccc}
-1.1 \varepsilon^{4} & 7.1 \varepsilon^{3} & 5.6 \varepsilon^{2} \\
7.1 \varepsilon^{3} & -6.2 \varepsilon^{2} & -0.10 \varepsilon \\
5.6 \varepsilon^{2} & -0.10 \varepsilon & -0.95
\end{array}\right) \mathrm{v}, \quad M_{D}=\left(\begin{array}{ccc}
-6.3 \varepsilon^{4} & 8.0 \varepsilon^{3} & -1.9 \varepsilon^{3} \\
-4.5 \varepsilon^{3} & 0.38 \varepsilon^{2} & -1.3 \varepsilon^{2} \\
0.88 \varepsilon^{2} & -0.23 \varepsilon & -0.51 \varepsilon
\end{array}\right) \mathrm{v}=M_{L}^{T}, \\
& M_{D N}=\left(\begin{array}{ccc}
h_{11}^{d n} \varepsilon^{3} & 0.21 \varepsilon^{2} & -2.7 \varepsilon \\
h_{21}^{d n} \varepsilon^{2} & -0.28 \varepsilon & -0.15 \\
h_{31}^{d n} \varepsilon^{2} & 2.1 \varepsilon & 0.086
\end{array}\right) \mathrm{v}, \quad M_{M N}=\left(\begin{array}{ccc}
-0.72 & -1.5 \varepsilon & h_{13}^{m n} \varepsilon^{2} \\
-1.5 \varepsilon & 0.95 \varepsilon^{2} & h_{23}^{m n} \varepsilon^{3} \\
h_{13}^{m n} \varepsilon^{2} & h_{23}^{m n} \varepsilon^{3} & 0.093
\end{array}\right) \Lambda_{R}, \\
& M_{v}=\left(\begin{array}{ccc}
-81 \varepsilon^{2} & -4.3 \varepsilon & 2.4 \varepsilon \\
-4.3 \varepsilon & -0.25 & 0.28 \\
2.4 \varepsilon & 0.28 & -1.1
\end{array}\right) \mathrm{v}^{2} / \Lambda_{R}
\end{aligned}
$$

Note that all prefactors except three in the above matrices are within a factor of 0.1 to 10 of unity for this best fit. For this best fit we find the neutrino mass values

$m_{1}=0, m_{2}=8.65, m_{3}=49.7 \mathrm{meV} ; \quad M_{1}=1.67 \times 10^{12}, M_{2}=6.85 \times 10^{13}, M_{3}=5.30 \times 10^{14} \mathrm{GeV}$

In addition, the best fit favors $\delta_{C P}=\pi$ for the leptonic CP Dirac phase. The value of $\varepsilon$ used for good fit convergence, $\varepsilon=1 / 6.5^{2}=0.0237$, then implies that the SU(12) GUT scale is about $M_{\mathrm{SU}(5)} / \varepsilon=8.4 \times 10^{17} \mathrm{GeV}$, just below the string scale, where we have used $2 \times 10^{16} \mathrm{GeV}$ for the $\mathrm{SU}(5)$ unification scale.

All remaining mass and mixing parameters are fit quite well by the model; however, since $M_{L}$ is just the transpose of $M_{D}$ in leading order in $\varepsilon$, the Georgi-Jarlskog relations [6] are not satisfied for the down quarks and charged leptons. We have checked that the addition of an adjoint $\mathbf{1 4 3}_{\mathrm{H}}$ Higgs field whose VEV points in the $B-L$ direction contributes to $M_{D}$ and $M_{L}$ at one higher order of $\varepsilon$, so that the $M_{L}=M_{D}^{T}$ relation is broken, and more accurate values can be obtained for the down quark and charged lepton mass eigenvalues.

\section{Summary}

A unified SU(12) SUSY GUT model was obtained by a brute force computer scan over many $\mathrm{SU}(12)$ anomaly-free sets of irreps containing $3 \mathrm{SU}(5)$ chiral families under the assumption that the symmetry is broken in stages from $\mathrm{SU}(12) \rightarrow \mathrm{SU}(5) \rightarrow \mathrm{SM}$. In doing so, looping over all $\mathrm{SU}(12)$ fermion and Higgs assignments was performed with good fits to the input data required. For this purpose an effective theory approach was used to determine the leading order tree-level diagrams for the dim- $(4+n)$ matrix elements in powers of $\varepsilon^{n}$, where $\varepsilon$ is the ratio of the $\mathrm{SU}(5)$ to the $\mathrm{SU}(12)$ scale. The best fit was obtained by requiring nearly all prefactors to be $\mathrm{O}(1)$, but the large number of them implies just a few predictions.

The SU(12) model considered is just one of many possibilities (including other assignments and larger $\mathrm{SU}(N)$ groups), but its features were among the most attractive found: Each $\mathrm{SU}(5)$ family supermultiplet can be assigned to a different $\mathrm{SU}(12)$ multiplet in the anomaly-free set. In 
the model considered, only one diagram appears for each matrix element for all 5 mass matrices, but some additional contribution is needed to obtain the Georgi-Jarlskog relations.

Among some distracting features we point out the prefactors are determined at the top quark scale. They should be run to the SU(5) unification scale to test their naturalness. The fit considers only real prefactors, so CP violation is not accommodated, but the fit preferred $\delta_{C P}=\pi$ over $\delta_{C P}=0$ for the leptonic CP phase. The complete breaking of $\mathrm{SU}(12) \rightarrow \mathrm{SU}(5)$ while preserving supersymmetry needs to be worked out in more detail and is under further study.

\section{Acknowledgments}

The work of RPF was supported by a fellowship within the Postdoc-Programme of the German Academic Exchange Service (DAAD). The work of RPF and TWK was supported by US DOE grant E-FG05-85ER40226. CHA thanks the Fermilab Theoretical Physics Department for its kind hospitality, where part of this work was carried out. Fermilab is operated by Fermi Research Alliance, LLC under Contract No. De-AC02-07CH11359 with the U.S. Department of Energy.

\section{References}

[1] C.H. Albright, R. Feger, and T.W. Kephart, Phys. Rev. D 86, (2012) 015012 [arXiv:1204.5471].

[2] R. Feger and T.W. Kephart, arXiv:1206.6379 [hep-ph].

[3] C.H. Albright, R. Feger, and T.W. Kephart, in proceedings of the CETUP $* 2012$ Workshop on Neutrino Physics and Unification, July 10 - August 1, 2012, Lead, South Dakota, arXiv:1210.-- [hep-ph].

[4] P.H. Frampton and T.W. Kephart, Phys. Rev. Lett. 48, (1982) 1237.

[5] P. Frampton and T. Kephart, Nucl. Phys. B211, (1983) 239.

[6] H. Georgi and C. Jarlskog, Phys. Lett. B 86, (1979) 297. 